\title{
РАЗДЕЛЕНИЕ ПАРАМЕТРА РАСТВОРИМОСТИ НА СОСТАВЛЯЮЩИЕ И ОПРЕДЕЛЕНИЕ КОЭФФИЦИЕНТОВ АКТИВНОСТИ САМОАССОЦИИРУЮЩИХСЯ ВЕЩЕСТВ ПО ДАННЫМ РАСПРЕДЕЛЕНИЯ МЕЖДУ ДВУМЯ ЖИДКИМИ ФАЗАМИ
}

Теория регулярных растворов в своей классической форме применима только для описания равновесия между неполярными компонентами. Тем не менее в последнее время появился ряд работ (например $\left[{ }^{1-8}\right]$ ), в которых эта теория в модифицированном виде распространена также на системы, где значительную долю общей энергии взаимодействия составляют вклады ориентационного взаимодействия и Н-связи. В этом случае используют дополнительные показатели (например дипольный момент, индекс Н-связи), учитывающие эти эффекты $[1,5,6]$, или из общей энергии взаимодействия молекул разделяют составляющие, обусловленные Н-связью и ориентацией диполей $[2-4,7,8]$. В настоящей работе сделана попытка использовать аналогичный подход для предсказания и корреляции свойств растворов самоассоциирующихся веществ в различных неполярных растворителях по данным распределения между двумя жидкими фазами.

\section{Распределение самоассоциирующихся веществ между фазами неполярных растворителей и воды}

Согласно развитой в $\left[{ }^{7}\right]$ концепции, энергию взаимодействия молекул в чистой жидкости можно рассматривать как сумму трех составляющих - энергии дисперсионного $\left(E_{d}\right)$ и полярного $\left(E_{p}\right)$ взаимодействия и энергии Н-связи $\left(E_{h}\right)$

$$
E=E_{d}+E_{p}+E_{h} .
$$

Суммарный параметр растворимости ( $\delta)$ определяется:

$$
\delta^{2}=\delta_{d}^{2}+\delta_{p}^{2}+\delta_{h}^{2},
$$

где $\delta=\left[\left(\Delta \mathrm{H}^{\text {иеп }}-R T\right) / V\right]^{0,5}, \quad \delta_{d}=\left(E_{d} / V\right)^{0,5}, \quad \delta_{p}=\left(E_{p} / V\right)^{0,5}, \quad \delta_{h}=\left(E_{h} / V\right)^{0,5}$, $\Delta \mathrm{H}^{\text {исп }}$ - энтальпия испарения, $V$ - мольный объем.

Для парциальной мольной энергии дисперсионного взаимодействия самоассоциирующегося вещества $A$ с неполярным растворителем $S$ можно напнсать

$$
\Delta E_{d A}=V_{A}\left(\delta_{d A}-\delta_{d S}\right)^{2}
$$


где индексы А и $\mathrm{s}$ относятся соответственно к растворенному веществу $A$ и растворителю $S$.

B растворах полярных веществ энергия полярного (диполь-дипольного) взаимодействия зависит как от момента постоянных $(\mu)$, так и индуцированных диполей $\left(\mu^{\text {пнд }}\right)$, которые частично взаимно компенси-

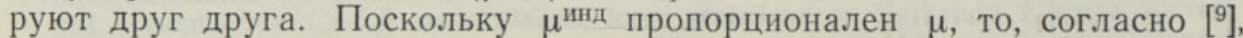
общая энергия полярного взаимодействия пропорциональна $\mu^{2}$ и для растворов $A$ в $S$ с $\delta_{p S}=0$ можно написать

$$
\Delta E_{p A}=V_{A}\left(C_{A} \mu_{A}-C_{S} \mu_{S}^{\mathrm{HH}}\right)^{2}=\psi V_{A} C_{A}^{2} \mu_{A}^{2}=\psi V_{A} \delta_{p A}^{2},
$$

где $C=f(\varepsilon, n, V), \psi=\left(1-\frac{C_{S} \mu_{S}^{\text {инд }}}{C_{A} \mu_{A}}\right)^{2}, \varepsilon-$ диэлектрическая проницаемость, $n$ - показатель преломления, $\psi$ - коэффициент, учитывающий поляризуемость растворителя за счет постоянных диполей вещества $A$. Для соединений одного гомологического ряда (с одинаковой поляризуемостью) $\psi$ должен быть постоянен, что действительно подтверждается результатами работ $[2,3,10,11]$.

Если растворитель не склонен образовывать с $A$ Н-связи и не самоассоциирован $\left(\delta_{h s}=0\right)$, то парциальная мольная энергия Н-связи выражается

$$
\Delta E_{h A}=V_{A} \delta_{h A}^{2} .
$$

Учитывая изменение парциальной мольной энтропии $\Delta S_{A}^{\text {см }}$ при смешении компонентов с различными мольными объемами, общую парциальную мольную энергию смешения вещества $A$ с растворителем $S$ можно написать в виде

$$
\Delta E_{A}^{\mathrm{cM}}=R T\left[\ln \frac{V_{A}}{V_{S}}+\left(1-\frac{V_{A}}{V_{S}}\right)\right]+V_{A}\left[\left(\delta_{d A}-\delta_{d S}\right)^{2}+\psi \delta_{p A}^{2}+\delta_{h A}^{2}\right] .
$$

Обозначим $\gamma_{A(S)}$ и $\gamma_{A(в)}-$ коэффициенты активности вещества $A$ соответственно в фазах $S$ и воды при бесконечном разбавлении, $K_{A}-$ коэффициент распределения $A$ между фазами $S$ и воды $\left(K_{A} \equiv x_{A(S)} / x_{A(\mathrm{~B})}\right.$ при $\left.x_{A(S)} \rightarrow 0\right), x-$ мольная доля. Тогда получим

$$
\ln \gamma_{A(\mathrm{~S})}=\ln \frac{V_{A}}{V_{S}}+\left(1-\frac{V_{A}}{V_{S}}\right)+\frac{V_{A}}{R T}\left[\left(\delta_{d A}-\delta_{d S}\right)^{2}+\psi \delta_{p A}^{2}+\delta_{h A}^{2}\right] .
$$

Учитывая, что

$$
\ln \gamma_{A(S)}=\ln \gamma_{A(\mathrm{~B})}-\ln K_{A},
$$

уравнение (7) можно переписать в виде

$$
\begin{aligned}
& \frac{R T}{V_{A}}\left[\ln K_{A}+\ln \frac{V_{A}}{V_{S}}+\left(1-\frac{V_{A}}{V_{S}}\right)\right]+\delta_{d S}^{2}= \\
= & \frac{R T}{V_{A}} \ln \gamma_{A(\mathrm{~B})}-\delta_{d A}^{2}-\psi \delta_{p A}^{2}-\delta_{h A}^{2}+2 \delta_{d A} \delta_{d S} .
\end{aligned}
$$


Обозначив

$$
\frac{R T}{V_{A}}\left[\ln K_{A}+\ln \frac{V_{A}}{V_{S}}+\left(1-\frac{V_{A}}{V_{S}}\right)\right]+\delta_{d S}^{2}=Y,
$$

$\frac{R T}{V_{A}} \ln \gamma_{A(\mathrm{~B})}-\delta_{d A}^{2}-\psi \delta_{p A}^{2}-\delta_{h A}^{2}=B_{0}$ и $2 \delta_{d A}=B_{1}$, получим

$$
Y=B_{0}+B_{1} \delta_{d S}
$$

т. е. для данного распределяющегося вещества зависимость $Y$ от $\delta_{d S}$ должна описываться параллельными прямыми с угловым коэффициентом $2 \delta_{d A}$. Расстояние между прямыми $(\Delta Y)$, соответствующими раство-

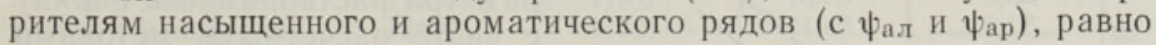

$$
\Delta Y=Y_{\text {ал }}-Y_{\text {ap }}=\left(\psi_{\text {ал }}-\psi_{\text {ap }}\right) \delta_{p A}^{2} .
$$

\section{Экспериментальная часть и обработка результатов}

Коэффициенты распределения полярных веществ при бесконечном разбавлении между растворителями (алканы, циклогексан, $\mathrm{CCl}_{4}$, ароматические углеводороды - всего 18 растворителей) и водой при $20^{\circ} \mathrm{C}$ определялись по методике, описанной нами ранее в [12]. Кроме оригинальных данных самих авторов использовались также данные из [13-16].

Мольные объемы твердых при температуре опыта веществ были рассчитаны по известным методам, исходя из результатов их сравнения, опубликованных нами в $\left[{ }^{17}\right]$.

Коэффициенты активности распределяющихся веществ в воде при бесконечном разбавлении, использованные для расчета коэффициентов $\psi$, определяли путем температурной экстраполяции данных в $\left[{ }^{18,19}\right]$ или рассчитывали по $\left[{ }^{18}\right]$ из взаимной растворимости жидкость-жидкость и равновесия твердое тело-жидкость.

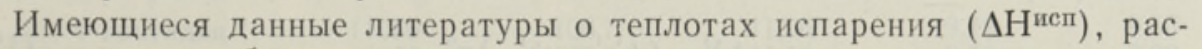
смотренных в работе веществ, получены разными авторами и по различным методам. Как правило, они плохо согласуются между собой. С целью уменьшения ошибок, вызванных многообразием методов, суммарный параметр растворимости полярных веществ $\left(\delta_{A}\right)$ был рассчитан из теплот испарения, найденных по данным $\left[{ }^{20-22}\right]$ из температурной зависимости давления паров. Более детально расчет теплот испарения будет описан в специальной статье.

Дисперсионную составляющую параметра растворимости полярных веществ $\left(\delta_{d A}\right)$ определяли двумя независимыми методами - из экспериментальных данных распределения между фазами растворителя и вод (I) и расчетным путем (II). По первому методу $\delta_{d A}$ рассчитывали из углового коэффициента $\left(B_{1}\right)$ прямых, описываемых зависимость $Y$ от $\delta_{d s}$ для данного вещества (уравнение $\left.(9)\right)$, используя в качестве критерия минимизации остаточную дисперсию функции $Y$ для двух параллельных прямых (одна для алканов, циклогексана и $\mathrm{CCl}_{4}$, другая для ароматических углеводородов; рис. 1). Для растворителей брали $\delta_{d S} \equiv \delta_{S}$. По второму методу для алкилпроизводных фенола и анилина и для двухатомных фенолов $\delta_{d A}$ рассчитывалась по углеводородному гомоморфу [23-25], для галогенпроизводных фенола и анилина и всех остальных соединений, для которых концепция гомоморфа неприменима, - по 
полуэмпирическому уравнению, выведенному в [ $\left.{ }^{4}\right]$ и связывающему $\delta_{d A}$ с показателем преломления $\left(n_{D}\right)$ :

$$
\delta_{d A}=-2,24+53 x-58 x^{2}+22 x^{3},
$$

где $x=\left(n_{D}^{2}-1\right) /\left(n_{D}^{2}+2\right)$.

Полученные результаты (табл. 1) показали, что экспериментальные и расчетные значения $\delta_{d A}$ хорошо согласуются между собой (средняя

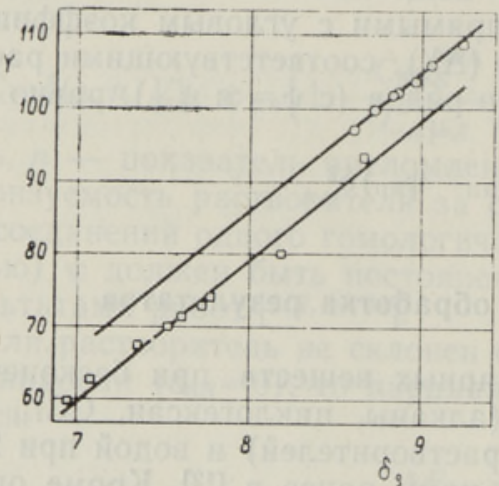

Рис. 1. Зависимость $Y$ от $\delta_{S}$ при распределении 4-этилфенола (экспериментальные данные из [16]).

$\square$ - алканы, циклогексан и $\mathrm{CCl}_{4}$;

О - ароматические углеводороды. погрешность 6,53\%). Таким образом, для определения дисперсионной составляющей параметра растворимости-наряду с чисто расчетными методами могут использоваться также экспериментальные данные о распределении между фазами.

Сопоставление значений $\Delta Y$, полученных по уравнению (10), с $\delta_{A}^{2}-\delta_{d A}^{2} \equiv \delta_{p A}^{2}+\delta_{h A}^{2}$ показывает, что между этими величинами существует почти строго пропорциональная зависимость c коэффициентом пропорциональности, равным 0,203 (стандартное отклонение для 38 точек 0,013 ) (рис. 2). Это означает, что для самоассоциирующихся веществ $\delta_{p A}^{2}$ и $\delta_{h A}^{2}$

в первом приближении можно также считать пропорциональными.

Обозначив $\delta_{h A}^{2} / \delta_{p A}^{2}=C,(\psi+C) /(1+C)=\psi$ и $\delta_{p A}^{2}+\delta_{h A}^{2}=\tau_{A}^{2}$, уравнение (10) можно записать

$$
\Delta Y=\left(\psi_{\mathrm{aJ}}^{\prime}-\psi_{\mathrm{ap}}^{\prime}\right) \tau_{\mathrm{A}}^{2} .
$$

Так как для ряда распределяющихся соединений известны коэффициенты активности в воде $\left(\gamma_{\text {A(в) }}\right)$, полученные независимым способом, то из данных о распределении определяли значения ф' для использованных растворителей. Для этого уравнение (8) преобразовали в форму

$$
Y+\frac{R T}{V_{A}} \ln \gamma_{A(\mathrm{~B})}+\delta_{d S}^{2}-\left(\delta_{d A}-\delta_{d S}\right)^{2}=\psi^{\prime} \tau_{A}^{2},
$$

откуда $\psi^{\prime}$ для данного растворителя определяли как среднее арифметическое нескольких значений, полученных с различными $A$ (табл. 2).

Полученные данные показывают, что для растворителей одного гомологического ряда значение $\psi$ ' действительно практически постоянно: взвешенное среднее для алифатических углеводородов, циклогексана и $\mathrm{CCl}_{4}$ (214 точек) $\psi_{\text {ал }}^{\prime}=0,402 \pm 0,085$, для ароматических углеводородов (159 точек) - $\psi_{\text {ap }}^{\prime}=0,196 \pm 0,057$.

Сопоставление экспериментальных и рассчитанных (с использованием приведенных выше величин $\delta_{d A}, \tau_{A}$ и $\left.\psi^{\prime}\right)$ значений $\lg \gamma_{A(в)}$ показы- 
Таблица 1

Теплоты испарения при температуре кипения, мольные объемы и параметры растворимости при $20^{\circ} \mathrm{C}$ для изученных соединений

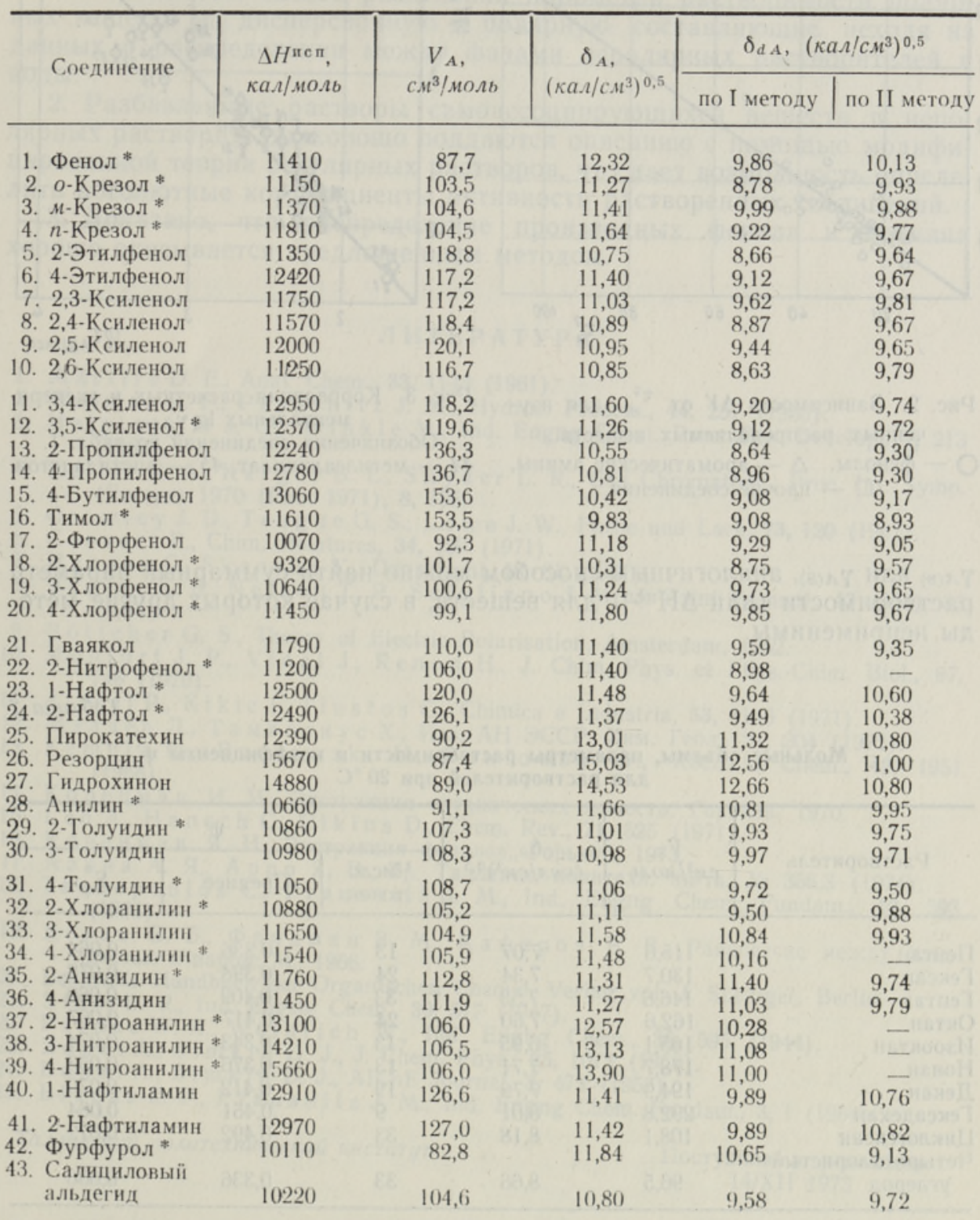

* Иепользовались для расчета ф'.

вает вполне удовлетворительное согласие (рис. 3). По мнению авторов, это дает право использовать изложенный подход также для предсказания коэффициентов активности других самоассоциирующихся веществ в неполярных растворителях, определив сначала из данных о распределении между фазами или расчетным путем $\delta_{d A}$ и $\tau_{A}$, после чего (исполь. зуя значения $\psi_{\mathrm{a},}^{\prime}$ и $\left.\psi_{\text {ap }}^{\prime}\right)$ легко определить $\gamma_{A(S)}$ или $\gamma_{A(\mathrm{~B})}$. Если известны 


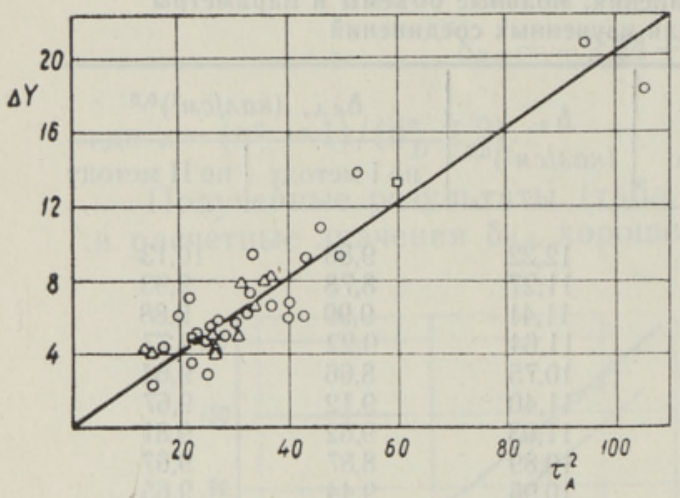

Рис. 2. Зависимость $\Delta Y$ от $\tau_{A}^{2}$ для изученных распределяемых веществ.

О - фенолы, $\triangle$ - ароматические амины, $\square$ - прочие соединения.

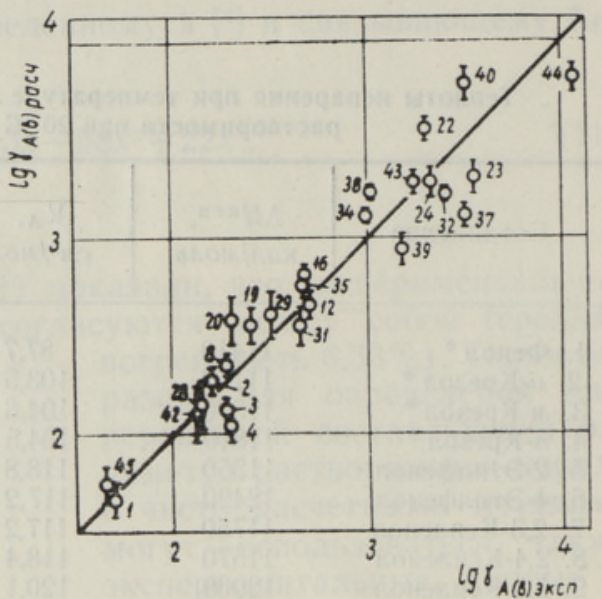

Рис. 3. Корреляция расчетных и экспериментальных $\lg \gamma_{A(\text { в) }}$.

Обозначения соединений по табл. 1.

44 - метилсалицилат, 45 - ацетилацетон.

$\gamma_{A(\text { в) }}$ или $\gamma_{A(S)}$, аналогичным способом можно найти суммарный параметр растворимости (или $\Delta \mathrm{H}^{\text {нсп) }}$ ) для веществ, в случае которых другие методы неприменимы.

Таблица 2

Мольные объемы, параметры растворимости и коэффициенты $\psi^{\prime}$ для растворителей при $20^{\circ} \mathrm{C}$

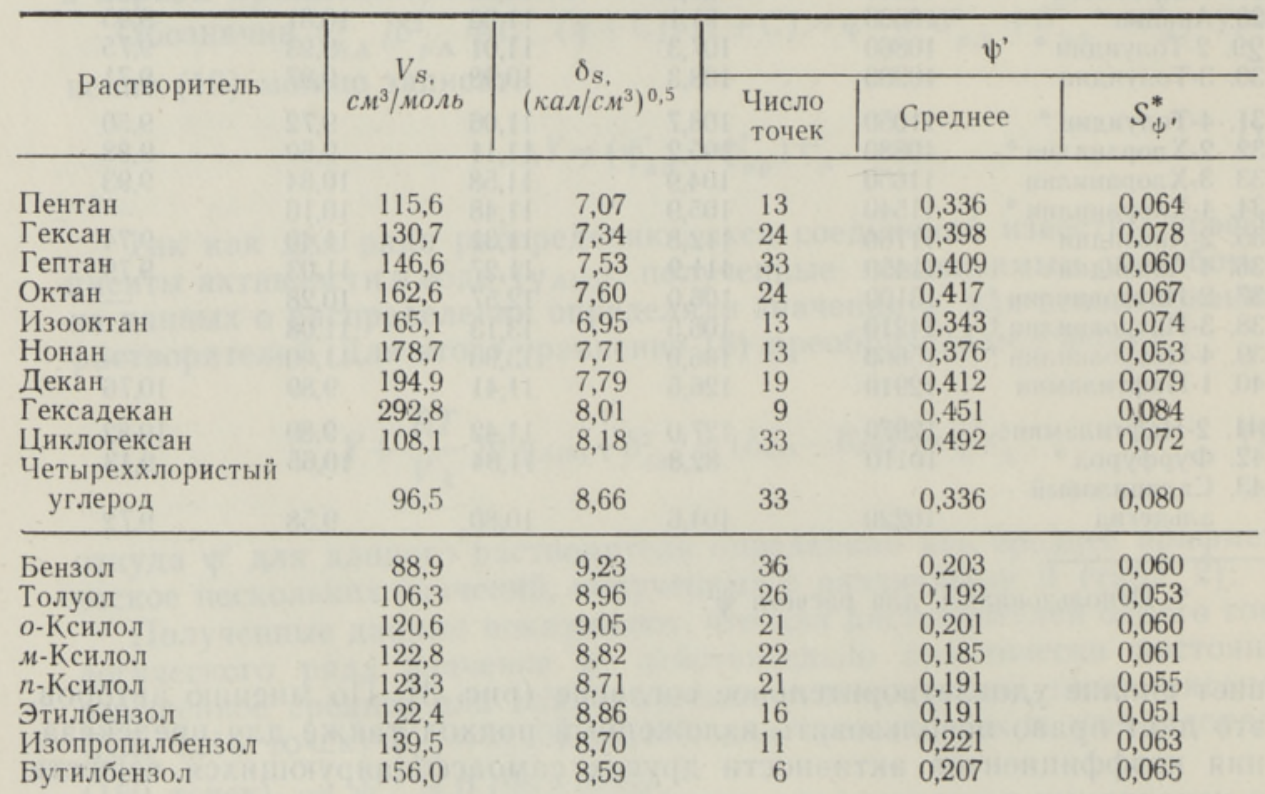

* Стандартное отклонение. 


\section{Выводы}

1. Предложен способ разделения параметра растворимости полярных веществ на дисперсионную и полярную составляющие исходя из данных о распределении между фазами неполярных растворителей и воды.

2. Разбавленные растворы самоассоциирующихся веществ в неполярных растворителях хорошо поддаются описанию с помощью модифицированной теории регулярных растворов, что дает возможность определить абсолютные коэффициенты активности растворенных соединений.

3. Доказано, что распределение производных фенола и анилина хорошо описывается предложенным методом.

\section{Л ИТЕРАТ У А}

1. M a rt ir e D. E., Anal. Chem., 33, 1143 (1961).

2. Weimer R. F., Prausnitz J. M., Hydroc. Process., 44, 237 (1965).

3. Helpinstill J. G., Winkle M., Ind. Engng Chem. Proc. Des. Develop., 7, 213 (1968).

4. Keller R. A., Karger B. L., S n y der L. R., Gas Chromatogr., Proc. Int. Symp. (Europe) 1970 (Pub. 1971), 8, 125.

5. Crowley J. D., T e a g u e G. S., L ow e J. W., Farbe und Lack, 73, 120 (1967).

6. C o s a e r t E., Chim. Peintures, 34, 169 (1971).

7. H a n sen C., Sk a r u p K., Danskkemi, 48, 81 (1967).

8. B a gley E. B., Nels on T. P., S c ig li a no J. M., J. Paint Technol., 43 (555), 35 (1971).

9. Böttch er G. S., Theory of Electric Polarisation, Amsterdam, 1952.

10. Monfort J. P., Vidal J., Renon H., J. Chim. Phys. et Phys.-Chim. Biol., 67, $748(1970)$.

11. Alessi P., Kikic I., Tlust os G., Chimica e Industria, 53, 1148 (1971).

12. Мэлде р Л., Т а м вели у х Х., Изв. АН ЭССР, Хим. Геол., 21, 204 (1972).

13. Kemula W., Buchowski H., Pawlowski W., Roczniki Chem., 42, 1951 (1968).

14. Коренман И. М., Экстракция органических веществ, Горький, 1970.

15. Le o A., H an sch C., El kins D., Chem. Rev., 71, 525 (1971).

16. Корен м ан Я. И., Экстракция фенолов, Горький, 1973.

17. А а рн а А. Я., А р ро Я. В., Тр. Таллинск. политехн. ин-та, № 356,3 (1974).

18. Tsonopoulos C., Prausnitz J. M., Ind. Engng Chem. Fundam., 10, 593 (1971).

19. Коган В. Б., Фридман В. М., Кафаров В. В., Равновесие между жидкостью и паром, М., 1966.

20. Beilsteins Handbuch der Organischen Chemie, Verlag von J. Springer, Berlin.

21. S tul1 D. R., Ind. Engng Chem., 39, 517 (1947).

22 P a rdee W. A., Weinrich W., Ind. Engng Chem., 36, 595 (1944).

23. B ond i A., S i mkin D. J., J. Chem. Phys., 25, 1073 (1956).

24. B on di A., S i m k in D. J., AIChE Journal, 3, 473 (1957).

25. Blanks R. F., Pra us nitz J. M., Ind. Engng Chem. Fundam., 3, 1 (1964).

Таллинский политехнический институт

Поступила в редакцию 14/XII 1973

\section{AUTOASSOTSIEERUVATE AINETE LAHUSTUVUSPARAMEETRI KOMPONENTIDE JA AKTIIVSUSTEGURI MAÄRAMINE FAASIDEVAHELISE JAOTUMISE ANDMETEL}

Esitatakse meetod autoassotsieeruvate ainete lahustuvusparameetri jaotamiseks dispersiooni- ja polaarsetest jõududest tingitud komponentideks, kasutades faasidevahelise tasakaalu andmeid. Seda rakendades saab arvutada jaotuvate ainete aktiivsustegureid tasakaalulistes faasides. Käsitlusviisi öigsust demonstreeritakse fenooli ja aniliini derivaatide näitel. 
A. AARNA, J. ARRO, L. MOLDER, H. TAMVELIUS

\section{DIVIDING THE SOLUBILITY PARAMETER INTO COMPONENTS AND DETERMINING THE ACTIVITY COEFFICIENTS FOR SELF-ASSOCIATED SUBSTANCES FROM THE LIQUID-LIQUID EQUILIBRIUM}

The possibility of calculating the infinite dilution activity coefficients for selfassociated compounds in nonpolar solvents and water has been shown. In this connection a method has been proposed for dividing the solubility parameter of a polar substance into contributions from dispersion and polar interactions on the ground of partition data between water and nonpolar solvents. This treatment has been verified in the case of phenols and aromatic amines. 